\title{
Editorial: Machine Learning and Big Data Analytics for loT-Enabled Smart Cities
}

\author{
Mian Ahmad Jan ${ }^{1} \cdot$ Xiangjian $\mathrm{He}^{2} \cdot$ Houbing Song ${ }^{3} \cdot$ Muhammad Babar $^{4}$ \\ Published online: 7 January 2021 \\ (C) Springer Science+Business Media, LLC, part of Springer Nature 2021
}

\section{Editorial}

Improving the cities is a persuasive global need due to the growth of world's population and a rapid increase in urbanization. It is expected that $67 \%$ of the world population will reside in cities by 2050. As urban population continues to swell due to high demand in migration on account of better job opportunities and improved quality of life, efficient delivery of services to citizens becomes paramount. The need for access to quality services and smarter communities is becoming increasingly important every passing day. All the major cities around the world are competing on the global stage for investment and harnessing the true potential of urbanization by eradicating extreme poverty and boost shared prosperity. As the smart cities continue to mature, specialized algorithms will be required to maintain the voluminous amount of data generated by these cities. The expansion of smart cities is resulting in the production of gigantic data at exceptional pace. Unfortunately, mostly produced data are washed away without pulling out useful knowledge and information due to the inadequacy of recognized standards and mechanisms. In addition, the dynamic environments of smart cities demand new machine learning techniques that are flexible to deal with the dynamic nature of data to achieve analytics and learn in real-time.

In this special issue, we received a total of 27 submissions. Upon rigorous review, seven high quality papers were selected.

Mian Ahmad Jan

mianjan@awkum.edu.pk

1 Department of Computer Science, Abdul Wali Khan University Mardan, Nowshera Road, College Chowk, Muslimabad, Mardan, Khyber Pakhtunkhwa 23200, Pakistan

2 School of Electrical and Data Engineering, University of Technology Sydney, 15 Broadway, Ultimo, NSW 2007, Australia

3 Department of Electrical, Computer, Software, and Systems Engineering, Embry-Riddle Aeronautical University, Daytona Beach, FL, USA

4 Iqra University, Islamabad, Pakistan
Among these papers, three papers are the extended versions of the paper accepted in "The 4th International Workshop on Securing IoT Networks (SITN 2020)" held under EAI Collaboratecom 2020. The remaining four papers were selected from open call. The first article titled "Machine learning based approach for sustainable social protection policies in developing societies" employed an unsupervised machine learning clustering technique for exploring big data that comprised of 88 attributes and 570 instances for better targeting of households that are in urgent need of welfare from the government. The unsupervised machine learning technique presented in this study is an efficient approach because it can be used by societies that are facing data constraints and can achieve optimal results for increasing the welfare of poor.

Sentiment analysis is gaining popularity in recent years as it is extremely useful for monitoring the social media platforms of smart cities and allows us to gain insight of public opinion about certain topics. The second article titled "Research on sentiment analysis of network forum based on BP neural network" proposed a neural network with specific objective of sentiment analysis. Compared with the current neural networks, the proposed approach has a shorter training time, which can effectively make up for the lack of emotional mechanism of the individuals in a smart city. This article uses large-scale emotional data set to carry out simulation experiments for sentiment analysis.

Energy consumption of a smart city's enterprise energy management system (EMS) is a complex process with nonlinearity, time-variance, larger delay, greater inertia and other dynamic characteristics, resulting in the failure of a single-item prediction model to achieve satisfactory prediction results. In the third article titled "BP Neural Network Combination Prediction for Big Data Enterprise Energy Management System", a combination prediction method based on Back Propagation (BP) neural network, was proposed to predict the energy consumption of EMS for improving the prediction accuracy. The energy consumption of enterprise energy management system (EMS) was predicted and analyzed using gray combination models, gray Markov chain, and BP neural network prediction model.

The fourth article titled "MapReduce-based Improved Random Forest Model for Massive Educational Data 
Processing and Classification" takes education data mining as the research theme, mine the existing massive education big data, compares the analysis methods of existing data models, and proposes an improved random forest reference model. The information gain of various features within a smart city ecosystem is calculated by introducing the feature weighting system, and the evaluation index is used to improve the existing data analysis.

The fifth article titled "Convolutional Neural Network for the Semantic Segmentation of Remote Sensing Images" applied Convolutional Neural Networks (CNN) on the semantic segmentation of remote sensing images. This paper improves the Encoder-Decoder CNN structure SegNet with index pooling and U-net to make them suitable for multi-targets semantic segmentation of remote sensing images. This paper also proposes an integrated algorithm for integrating these two models.

The sixth article titled "Mining of High-Utility Patterns in Big IoT-based Databases" introduces Potential high-utility itemset mining (PHUIM) for revealing valuable patterns in an uncertainty database. Unfortunately, even though the previous methods are all very effective and powerful to mine, the potential high-utility item sets quickly. These algorithms are not specifically designed for a database with an enormous number of records. In this work, a dataset is assumed to be too big to be loaded directly into memory and be duplicated or modified. A MapReduce framework is proposed that can be used to handle these types of situations. This article aims to reduce the frequency of dataset scans while still maximizing the parallelization of all processes.

The last paper titled "A Comprehensive Survey on Machine Learning-based Big Data Analytics for IoT-enabled Smart Healthcare System" presented a novel and comprehensive review on the application of machine learning techniques for big data analysis in the smart healthcare sector. The strength and weaknesses of existing techniques along with various research challenges are highlighted. This paper also provide an insight for healthcare practitioners and government agencies to keep themselves well-equipped with the latest trends in Machine Learning-enabled big data analytics for smart healthcare. Finally, this paper thoroughly investigated the emerging and cross-domain architectures of IoT, i.e., Internet of Nano-Things (IoNT), and web of Things (WoT), to make a universal IoT vision a reality: a vision that successfully integrates this technology in almost all domains and that will hopefully flourish our daily lives in the years to come.

Acknowledgements The guest editors are thankful to reviewers for their effort in reviewing the manuscripts. We also thank the Editor-in-Chief, Prof. Imrich Chlamtac for his supportive guidance during the entire process. He has been extremely helpful during the entire process. We are also thankful to the wonderful and highly professional team of EAI associated with special issues and conferences. They have been extremely helpful and their timely responses and guidance has an immense impact on the management of this special issue. We are also thankful to the authors for their contributions and for taking into account the comments of reviewers to bring their papers up to a very high standard demanded by Springer Mobile Networks and Applications.

Publisher's note Springer Nature remains neutral with regard to jurisdictional claims in published maps and institutional affiliations.

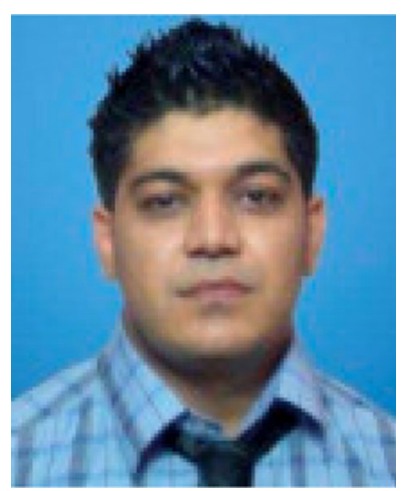

Mian Ahmad Jan is an assistant professor at the department of computer science, Abdul Wali Khan University Mardan, Pakistan. He completed his $\mathrm{PhD}$ at the University of Technology Sydney (UTS), Australia. He had been the recipient of various prestigious scholarships during his $\mathrm{PhD}$ studies. He was the recipient of International Research Scholarship (IRS), UTS and Commonwealth Scientific Industrial Research Organization (CSIRO) scholarships. He has been awarded the best researcher awarded for the year 2014 at the University of Technology Sydney Australia. His research interests include energy-efficient and secured communication in Wireless Sensor Networks and Internet of Things. Recently, he has been actively involved in machine learning, big data analytics, smart cities infrastructure and vehicular ad hoc networks. His research has been published in prestigious journals and conferences. He has been guest editor of numerous special issues in various prestigious journals and has edited numerous books.

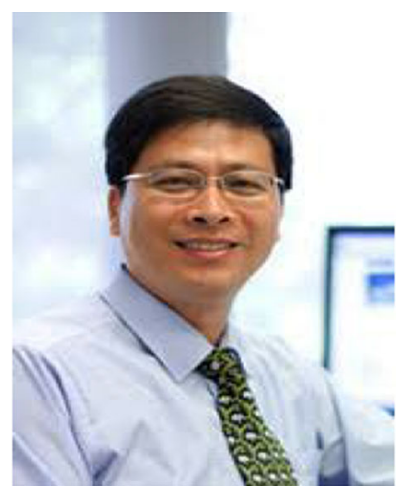

Xiangjian $\mathrm{He}$ is a Professor of School of Electrical and Data Engineering, University of Technology Sydney, Australia. $\mathrm{He}$ has received various research grants including four national Research Grants awarded by Australian Research Council (ARC). He has played various chair roles in many international conferences such as ACM MM, $\mathrm{MM} \mathrm{M}$, I C D A R, I E E E BigDataSE, IEEE CIT, IEEE AVSS, IEEE TrustCom, IEEE ICPR and IEEE ICARCV. In recent years, he has many high quality publications in prestigious journals. He has presented invited keynote speeches in over 15 international conferences, including several top ranked international conferences, since 2008. He has recently been involved in editing for various international journals. He is in the editorial boards of seven international journals. 


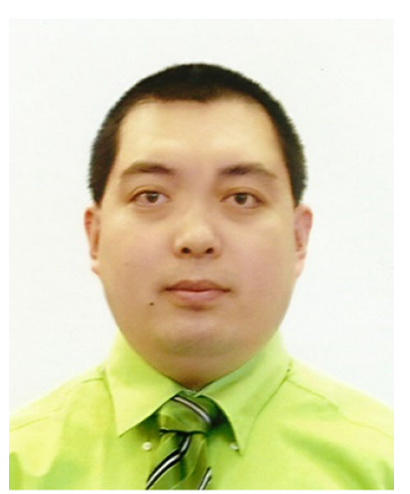

Houbing Song received the Ph.D. degree in electrical engineering from the University of Virginia, Charlottesville, VA, in August 2012. In August 2017, he joined the Department of Electrical, Computer, Software, and Systems Engineering, Embry-Riddle Aeronautical University, Daytona Beach, FL, where he is currently an Assistant Professor and the Director of the Security and Optimization for Networked Globe Laboratory (SONG Lab, www.SONGLab.us). He serves as an Associate Technical Editor for IEEE Communications Magazine. He is the editor of four books and author of more than 100 articles. His research interests include cyberphysical systems, internet of things, cybersecurity and privacy, edge computing, big data analytics, and wireless communications and networking. $\mathrm{He}$ is a senior member of IEEE and ACM.

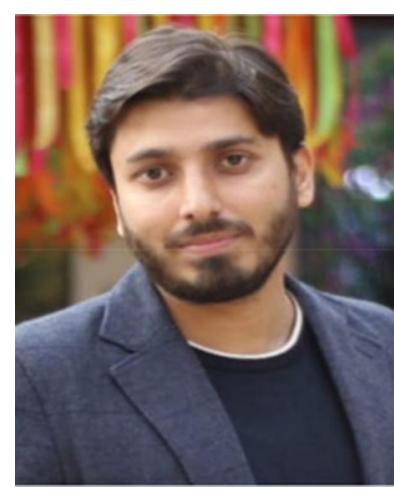

Muhammad Babar completed his PhD from National University of Sciences and Technology (NUST), Islamabad, Pakistan in 2018. He is currently serving as an Assistant Professor at Iqra University, Islamabad, Pakistan. His research area includes but not limited to Big Data Analytics, Internet of Things (IoT), Smart City Design and Planning, and Social Web of Things (SWOT). He has published his research work in topranked various Elsevier, IEEE and ACM journals. He also presented his research work in leading International conferences held in USA. He has been an active reviewer for Elsevier FGCS, Springer MONET, Elsevier Computer Networks, Sensor Systems, and IEEE Access. He also has been a part of the organizing committee of international conference. 